\title{
Remarks on the Continuity of the Local Minimizer of Scalar Integral Functionals With Nonstandard General Growth Conditions
}

\author{
Dedicated to Caterina and Delia Granucci \\ Tiziano Granucci ${ }^{1}$ \\ ${ }^{1}$ Instituto P. Calamandrei, Italy \\ Correspondence: Tiziano Granucci, Instituto P. Calamandrei, Italy. E-mail: tizianogranucci@libero.it \\ Received: July 28, 2014 Accepted: September 28, 2014 Online Published: October 9, 2014 \\ doi:10.5539/jmr.v6n4p1 URL: http://dx.doi.org/10.5539/jmr.v6n4p1 \\ I thank my family, particularly Elisa, Caterina, Delia, Laura and Giuliano. I thank my colleagues and frends of \\ the Istituto P. Calamandrei, particulary Cristina, Ilaria, Matteo, Sandra, Silvana and Stefania. A last thanks goes \\ to Michele and Massimo for their compluter support.
}

\section{Abstract}

In this paper we show a regularity theorem for local minima of scalar integral functionals of the Calculus of Variations with nonstandard general growth conditions. Let us consider functionals in the following form

$$
\mathcal{F}[u, \Omega]=\int_{\Omega} f(x, u(x), \nabla u(x)), d x
$$

where $f: \Omega \times \mathbb{R} \times \mathbb{R}^{N} \rightarrow \mathbb{R}$ is a Carathéodory function satisfying the inequalities

$$
\Phi(|z|)-c_{1} \leq f(x, s, z) \leq c_{2}\left[1+\left(\Phi^{*}(|z|)\right)^{\beta}+\left(\Phi^{*}(|s|)\right)^{\beta}\right]
$$

for each $z \in \mathbb{R}^{N}, s \in \mathbb{R}$ and for $\mathcal{L}^{N}$-a. e. $x \in \Omega$, where $c_{1}$ and $c_{2}$ are two positive real constants, with $c_{1}<c_{2}, \Omega$ is an open subset of $\mathbb{R}^{N}, N \geq 2, \Phi \in \Delta_{2}^{m} \cap \nabla_{2}^{r}$ [Definition 6 and Definition 8], $1 \leq r<m<N$ and the function $\Phi^{*}$ is the Sobolev conjugate of $\Phi$ [Definition 12], $\beta$ is a positive real number that we will opportunely fix [Hypothesis $\left.H_{1, f}\right]$.

Keywords: variational inequality, regularity, Hölder continuity, nonstandard growth conditions

AMS Classification: [2010] Primary 35B65; Secondary 49J45, 49 J40

\section{Introduction}

In this paper we show a regularity theorem for local minima of scalar integral functionals of the Calculus of Variations with nonstandard general growth conditions.

Let us consider functionals in the following form

$$
\mathcal{F}[u, \Omega]=\int_{\Omega} f(x, u(x), \nabla u(x)) d x
$$

where $f: \Omega \times \mathbb{R} \times \mathbb{R}^{N} \rightarrow \mathbb{R}$ is a Carathéodory function satisfying the inequalities

$$
\Phi(|z|)-c_{1} \leq f(x, s, z) \leq c_{2}\left[1+\left(\Phi^{*}(|z|)\right)^{\beta}+\left(\Phi^{*}(|s|)\right)^{\beta}\right]
$$

for each $z \in \mathbb{R}^{N}, s \in \mathbb{R}$ and for $\mathcal{L}^{N}$-a. e. $x \in \Omega$, where $c_{1}$ and $c_{2}$ are two positive real constants, with $c_{1}<c_{2}$, $\Omega$ is an open subset of $\mathbb{R}^{N}, N \geq 2, \Phi \in \triangle_{2}^{m} \cap \nabla_{2}^{r}$ [Definition 6 and Definition 8], $1 \leq r<m<N$ and the function $\Phi^{*}$ is 
the Sobolev conjugate of $\Phi$ [Definition 12], $\beta$ is a positive real number that we will opportunely fix [Hypothesis $\left.H_{1, f}\right]$. The research of regularity results for weak solutions of elliptic and parabolic PDEs starts from the basic results of De Giorgi (1957) and Nash (1958). In 1990s a remarkable production of regularity results for functionals with general growths has been developed.

In 1996, Mascolo and Papi have determined an Harnack inequality for the minimizer of the functional (1.1) under the following conditions: $f(z)=\Phi(|z|)$ where $\Phi$ is a N-function and $\Phi \in \Delta_{2} \cap \nabla_{2}$. We observe that $\Phi \in \Delta_{2} \cap \nabla_{2}$ implies

$$
c_{3} t^{p}-c_{4}<\Phi(t)<c_{5} t^{m}+c_{6} \quad \text { for } t>0
$$

with real positive constants $c_{3}, c_{4}, c_{5}, c_{6}$ and $1<p \leq m$. A classical regularity theorem for functionals with standard growth conditions $(p=m)$ has been proved in (De Giorgi, 1957) (we refer also to Giaquinta \& Giusti, 1984; Giusti, 1994). In 1991, Moscariello and Nania have obtained a results of Hölder continuity for the localminima of functional of the type (1.1) under the following hypothesis, $f(z)=\Phi(|z|)$ where $\Phi$ is a convex and increasing function, $\Phi \in \Delta_{2}$ and (1.2) holds with $1<p \leq m<\frac{N p}{N-p}$. In 1991, Lieberman proved an Harnack inequality for the minimizer of the functional (1.1) with $\Phi \in C^{2}$ suth that

$$
c_{7} \leq \frac{t \ddot{\Phi}(t)}{\dot{\Phi}(t)} \leq c_{8} \quad \text { for } t>0
$$

with $0<c_{7}<c_{8}$. In (Granucci, 2006, 2014a, 2014b), the author has extended, partially, the precedents results. Moreover in 1994, Klimov studies this problem when $\Phi$ satisfies $\nabla_{2}$ but not a $\Delta_{2}$ condition.

In this paper we proof a theorem on the regulaity of quasi-minima of the functional $\mathcal{F}[u, \Omega]$ with the following hypotheses.

$\left[\mathbf{H}_{0, f}\right] \quad$ Let $\Phi \in \triangle_{2}^{m} \cap \nabla_{2}^{r}$ be a N-function and $1 \leq r \leq m<N$.

$\left[\mathbf{H}_{1, f}\right] \quad f: \Omega \times \mathbb{R} \times \mathbb{R}^{N} \rightarrow \mathbb{R}$ is a Carathéodory function satisfying the inequalities

$$
\Phi(|z|)-c_{1} \leq f(x, s, z) \leq c_{2}\left[1+\left(\Phi^{*}(|z|)\right)^{\beta}+\left(\Phi^{*}(|s|)\right)^{\beta}\right]
$$

for each $z \in \mathbb{R}^{N}, s \in \mathbb{R}$ and for $\mathcal{L}^{N}$-a. e. $x \in \Omega$, where $c_{1}$ and $c_{2}$ are two positive real constants, with $c_{1}<c_{2}, \Omega$ is an open subset of $\mathbb{R}^{N}, N \geq 2, \Phi \in \triangle_{2}^{m} \cap \nabla_{2}^{r}$ and the function $\Phi^{*}$ is the Sobolev conjugate of $\Phi$. Let us define

$$
\Gamma_{1}(N, m, r, \beta)=\alpha_{1} N+\left[\alpha_{1} N+\left(N-m^{*} \beta\right)\left(1+\alpha_{2}\right)\right] \vartheta \alpha_{1}-m^{*} \beta+\left(N-m^{*} \beta\right) \alpha_{2}
$$

where $\alpha_{1}=\frac{[N-(1-\beta) m] r-N m \beta}{(N-m) r}, \alpha_{2}=\frac{\beta m N}{(N-m) r}-1, m^{*}=\frac{m N}{N-m}$ and $\vartheta$ is the positive solution of $\vartheta(\vartheta+1)=\alpha_{1}$, i.e. $\vartheta=\frac{-1+\sqrt{1+4 \alpha_{1}}}{2}$. Let us consider the system

$$
\left\{\begin{array}{l}
1 \leq r \leq m<N \\
\frac{r(N-m)}{m N}<\beta<\min \left(\frac{r(N-m)}{m(N-r)}, \frac{N-m}{m}\right) \\
\alpha_{1} N+\left(N-m^{*} \beta\right)\left(1+\alpha_{2}\right) \leq N \\
\alpha_{1} N+\left[\alpha_{1} N+\left(N-m^{*} \beta\right)\left(1+\alpha_{2}\right)\right] \vartheta \alpha_{1}-m^{*} \beta+\left(N-m^{*} \beta\right) \alpha_{2} \geq 0
\end{array}\right.
$$

then for every $r$ and $m$, with $1 \leq r \leq m<N$, there exists $\beta_{1, r, m, N}$, implicitly defined by $\Gamma_{1}(N, m, r, \beta)=0$, such that if $\frac{r(N-m)}{m N}<\beta<\beta_{1, r, m, N}$, then $\beta$ is a solution of the system (1.4). Our hypoteses on $\beta$ will be

$$
\frac{r(N-m)}{m N}<\beta<\beta_{1, r, m, N}
$$

$\mathbf{H}_{2, f} \quad$ For every $t \geq 0$ we have

$$
t^{r} \leq \Phi(t) .
$$

Remark 1 For the whole paper we will suppose that the system (1.4) has some solutions, in Appendix we will study in detail such system and we will find some conditions of existence, the relationship (1.5).

Remark 2 If $r=m$ then $\Phi(t) \sim t^{r}$ [Definition 4] and $\Phi^{*}(t) \sim t^{r^{*}}$ [Definition 12 and Lemma 3], this case has been studied by the author in (Granucci, submitted) and with more restrictive hypotheses in (Moscariello \& Nania, 1991). 
Remark 3 The hypothesis $\mathrm{H}_{2, f}$ is purely tecnical, it can be removed [Corollary 1].

Moreover we will assume that $f$ satisfies on of the following hypotheses.

$\mathbf{H}_{3,1, f}$ For almost every $x \in \Omega f(x, \cdot, \cdot)$ is a convex function.

$\mathbf{H}_{3,2, f}$ For almost every $x \in \Omega$ and for every $s \in \mathbb{R}, f(x, s, \cdot)$ is a convex function; moreover there exists a constant $c_{3}>0$ such that

$$
f\left(x, s_{1}, \xi\right) \leq c_{3} f\left(x, s_{2}, \xi\right)
$$

for almost every $x \in \Omega$, for every pair $s_{1}, s_{2} \in \mathbb{R}$, with $\left|s_{1}\right| \leq\left|s_{2}\right|$, and for every $\xi \in \mathbb{R}^{N}$.

Definition 1 If $u \in V=\left\{v \in W^{1,1}(\Omega): \mathcal{F}[u, \Omega]<+\infty\right\}$ then $u$ is a local minimizer of $\mathcal{F}[u, \Omega]$ if

$$
\mathcal{F}[u, \operatorname{supp}(\varphi)] \leq \mathcal{F}[u+\varphi, \operatorname{supp}(\varphi)]
$$

for every $\varphi \in W^{1,1}(\Omega)$ with $\operatorname{supp}(\varphi) \subset \subset \Omega$.

Our principal result is the following theorem.

Theorem 1 (Main Theorem) Let $u \in V$ be a local minimizer of $\mathcal{F}[u, \Omega]$, with $f$ satisfying conditions $H_{0, f}$, $H_{1, f}$, $H_{2, f}, H_{3,1, f}$ or $H_{0, f}, H_{1, f}, H_{2, f}, H_{3,2, f}$; then $u$ is locally Hölder continuous in $\Omega$.

The precedent Theorem 1 extends the results gotten in (Dall'Aglio, Mascolo and Papi, 1997, 1998).

Corollary 1 Let $u \in V$ be a local minimizer of $\mathcal{F}[u, \Omega]$, with $f$ satisfying conditions $H_{0, f}, H_{1, f}, H_{3,1, f}$ or $H_{0, f}$, $H_{1, f}, H_{3,2, f}$; then $u$ is locally Hölder continuous in $\Omega$.

Proof. Using Lemma 2 (iii) there exists a N-function $\Phi_{1} \sim \Phi$ such that $\Phi_{1}$ satisfys $H_{2, f}$. Moreover, since $\Phi_{1} \sim \Phi$, there exist some positive constants $\varkappa_{1}, \varkappa_{2}$ and $t_{0}$ such that

$$
\Phi\left(\varkappa_{1} t\right) \leq \Phi_{1}(t) \leq \Phi\left(\varkappa_{2} t\right)
$$

for all $t>t_{0}$. If $0 \leq t \leq t_{0}$, since $\Phi_{1}$ and $\Phi$ are increasing and continuous functions, we have

$$
\Phi\left(\varkappa_{1} t\right)-c_{10} \leq \Phi_{1}(t) \leq \Phi\left(\varkappa_{2} t\right)+c_{11}
$$

where $c_{10}$ and $c_{11}$ are positive constants. Using (1-9) and (1.10) it follows

$$
\Phi\left(\varkappa_{1} t\right)-c_{12} \leq \Phi_{1}(t) \leq \Phi\left(\varkappa_{2} t\right)+c_{13}
$$

for all $t \geq 0$. Let us consider $\Phi_{2}(t)=\Phi\left(\varkappa_{1} t\right)$, since $\Phi$ fulfils the hypotesis $H_{0, f}$ and $H_{3,1, f}$, then $\Phi_{2}$ fulfils the hypothesis $H_{0, f}$ and $H_{3,1, f}$; using (1.2) and Lemma 1 (i) we get

$$
\begin{aligned}
\Phi_{2}(|z|)-c_{14} & \leq f(x, s, z) \\
& \leq c_{15}\left[\left(\frac{\varkappa_{2}}{\varkappa_{1}}\right)^{\beta m^{*}}\left(\Phi^{*}(|z|)\right)^{\beta}+\left(\frac{\varkappa_{2}}{\varkappa_{1}}\right)^{\beta m^{*}}\left(\Phi^{*}(|s|)\right)^{\beta}+1\right] \\
& \leq c_{15}\left(\frac{\varkappa_{2}}{\varkappa_{1}}\right)^{\beta m^{*}}\left[\left(\Phi^{*}(|z|)\right)^{\beta}+\left(\Phi^{*}(|s|)\right)^{\beta}+1\right] .
\end{aligned}
$$

Corollary 1 follows using (1.12) and Theorem 1.

Particulary Theorem 1 and Corollary 1 can be applied in the following cases

$$
\begin{array}{ll}
\Phi(t)=t^{p} & \text { with } p>1, \\
\Phi(t)=t^{p} \ln ^{a}(1+t) & \text { with } p \geq 1 \text { and } a>0, \\
\Phi(t)= \begin{cases}t^{p} & \text { if } 0<t<t_{0} \\
e^{\left(\frac{p+q}{2}+\frac{q-p}{2} \sin (\ln (\ln (t)))\right) \ln (t)} & \text { if } t \geq t_{0}\end{cases} & \text { where } \sin \left(\ln \left(\ln \left(t_{0}\right)\right)\right)=-1 \text { and } 1<p<q<N .
\end{array}
$$

\section{Definitions}

Definition 2 A continuous and convex function $\Phi:[0,+\infty) \rightarrow[0,+\infty)$ is called $N$-function if it satisfies

$$
\begin{aligned}
& \Phi(0)=0 \text { and } \Phi(t)>0 \text { if } t>0 ; \\
& \lim _{t \rightarrow 0^{+}} \frac{\Phi(t)}{t}=0 ; \\
& \lim _{t \rightarrow+\infty} \frac{\Phi(t)}{t}=+\infty .
\end{aligned}
$$


For exemple the function $\Phi_{p, \beta}(t)=t^{p} \ln ^{\beta}(1+t)$ for $p>1$ and $\beta \geq 0$ or $p=1$ and $\beta>0$ is a $\mathrm{N}$-function. Actually, only the growth at infinity really matters in the definition of $\mathrm{N}$-function. Indeed, given a continuous and convex function $A:[0,+\infty) \rightarrow[0,+\infty)$ satisfying

$$
\lim _{t \rightarrow+\infty} \frac{A(t)}{t}=+\infty
$$

there exist a $\mathrm{N}$-function $\Phi$ and $t_{0}>0$ such that for every $t>t_{0}$ there holds

$$
A(t)=\Phi(t) .
$$

The function $A$ is called principal part of the $\mathrm{N}$-function $\Phi$. For exemple there exists a $\mathrm{N}$-function $\Phi$ such that $\Phi(t)=t^{\ln (t)}$ near infinity or there exists a $\mathrm{N}$-function $\Phi$ such that $\Phi(t)=t \ln (t)$ near infinity.

Definition 3 If $\Phi_{1}$ and $\Phi_{2}$ are two N-functions we say that $\Phi_{1}$ dominates $\Phi_{2}$ near infinity if there exist two positive constants $\varkappa$ and $t_{0}$ such that

$$
\Phi_{2}(t) \leq \Phi_{1}(\varkappa t)
$$

for all $t \geq t_{0}$.

Definition 4 If $\Phi_{1}$ and $\Phi_{2}$ are two N-functions we say that $\Phi_{1}$ and $\Phi_{2}$ are equivalent near infinity $\left(\Phi_{1} \sim \Phi_{2}\right)$ if and only if there exist some positive constants $\varkappa_{1}, \varkappa_{2}$ and $t_{0}$ such that

$$
\Phi_{1}\left(\varkappa_{1} t\right) \leq \Phi_{2}(t) \leq \Phi_{1}\left(\varkappa_{2} t\right)
$$

for all $t \geq t_{0}$.

Remark 4 If $0<\lim _{t \rightarrow+\infty} \frac{\Phi_{1}(t)}{\Phi_{2}(t)}<+\infty$ then $\Phi_{1}$ and $\Phi_{2}$ are equivalent near infinity. Let us introduce two important classes of $\mathrm{N}$-functions.

Definition 5 A N-function $\Phi$ is of class $\Delta_{2}$ globally in $(0,+\infty)$ if exists $k>1$ such that

$$
\Phi(2 t) \leq k \Phi(t) \quad \forall t \in(0,+\infty) .
$$

Definition 6 A convex function $\Phi$ is of class $\Delta_{2}^{m}$ globally in $(0,+\infty)$, with $m \geq 1$, if for every $\lambda>1$

$$
\Phi(\lambda t) \leq \lambda^{m} \Phi(t) \quad \forall t \in(0,+\infty) .
$$

Remark 5 The class $\triangle_{2}^{1}$ contains only linear functions.

Definition $7 \mathrm{~A}$ N-function $\Phi$ is of class $\nabla_{2}$ globally in $(0,+\infty)$ if exists $l>1$ such that

$$
\Phi(t) \leq \frac{\Phi(l t)}{2 l} \quad \forall t \in(0,+\infty) .
$$

Definition 8 A convex function $\Phi$ is of class $\nabla_{2}^{r}$ globally in $(0,+\infty)$, with $r \geq 1$, if for every $\lambda>1$

$$
\lambda^{r} \Phi(t) \leq \Phi(\lambda t) \quad \forall t \in(0,+\infty) .
$$

Remark 6 Every N-functions belongs to $\nabla_{2}^{1}$.

Remark 7 We observe that

$$
\Delta_{2}=\bigcup_{m>1} \Delta_{2}^{m}
$$

and

$$
\nabla_{2}=\bigcup_{r>1} \nabla_{2}^{r}
$$

The $\mathrm{N}$-functions $\Phi \in \Delta_{2}^{m}$ are characterized by the following result.

Lemma 1 Let $\Phi$ be a $N$-function and let $\dot{\Phi}_{-}$be its left derivative. For $m \geq 1$ the following properties are equivalent:

(i) $\Phi(\lambda t) \leq \lambda^{m} \Phi(t)$, for every $t \geq 0$, for every $\lambda>1$;

(ii) $t \dot{\Phi}_{-}(t) \leq m \Phi(t)$, for every $t \geq 0$;

(iii) the function $\frac{\Phi(t)}{t^{m}}$ is nonincreasing on $(0,+\infty)$. 
The N-functions $\Phi \in \nabla_{2}^{r}$ are characterized by the following result.

Lemma 2 Let $\Phi$ be a $N$-function and let $\dot{\Phi}_{-}$be its left derivative. For $r \geq 1$ the following properties are equivalent:

(i') $\Phi(\lambda t) \geq \lambda^{r} \Phi(t)$, for every $t \geq 0$, for every $\lambda>1$

(ii') $t \dot{\Phi}_{-}(t) \geq r \Phi(t)$, for every $t \geq 0$;

(iii') the function $\frac{\Phi(t)}{t^{r}}$ is nondecreasing on $(0,+\infty)$.

Remark 8 If $\Phi \in \triangle_{2}^{m}$ using Lemma 1 (iii) it follows

$$
\Phi(t) \leq c_{16} t^{m}
$$

for every $t>1$.

Remark 9 If $\Phi \in \nabla_{2}^{r}$ using Lemma 2 (iii) it follows

$$
\Phi(t) \geq c_{17} t^{r}
$$

for every $t>1$.

Remark 10 Moreover, if $\Phi \in \Delta_{2}^{m} \cap \nabla_{2}^{r}$ then

$$
c_{17} t^{r} \leq \Phi(t) \leq c_{16} t^{m}
$$

for every $t>1$.

Remark 11 If $\Phi$ is a $\mathrm{N}$-function of class $\triangle_{2}^{m}$ globally in $(0,+\infty)$, then we have $\Phi(\lambda t) \leq \lambda^{m} \Phi(t)$ for every $t \in(0,+\infty)$ and $\lambda>1$. Let us put $t=\frac{s}{\lambda}$ then we have $\frac{\Phi(s)}{\lambda^{m}} \leq \Phi\left(\frac{s}{\lambda}\right)$ and $\Phi^{-1}\left(\frac{\Phi(s)}{\lambda^{m}}\right) \leq \frac{s}{\lambda}$ for every $s \in(0,+\infty)$ and $\lambda>1$. Let us put $s=\Phi^{-1}(w)$ then we have $\Phi^{-1}\left(\frac{w}{\lambda^{m}}\right) \leq \frac{\Phi^{-1}(w)}{\lambda}$ for every $w \in(0,+\infty)$ and $\lambda>1$. Let us put $\frac{1}{\lambda^{m}}=a$ then we have $\Phi^{-1}(a w) \leq a^{\frac{1}{m}} \Phi^{-1}(w)$ for every $w \in(0,+\infty)$ and $a \in(0,1)$.

Definition 9 We say that the N-function $\Phi$ satisfies the $\Delta^{\prime}$ - condition if there exist positive constants $c_{9}$ and $t_{0}$ such that

$$
\Phi(t s) \leq c_{9} \Phi(t) \Phi(s)
$$

for every $t, s \geq t_{0}$. If $t_{0}=0$ we say that $\Phi$ satisfies globally the $\Delta^{\prime}-$ condition $\left(\Phi \in \Delta^{\prime}\right.$ in $\left.(0,+\infty)\right)$.

Let us consider the $\mathrm{N}$-functions

$$
\begin{array}{ll}
\Phi_{1}(t)=t^{p} & \text { with } p>1 \\
\Phi_{2}(t)=t^{p}(|\ln (t)|+1) & \text { with } p>1 \\
\Phi_{3}(t)=(1+t) \ln (1+t)-t ; & \\
\Phi_{4}(t)=\frac{t^{2}}{1+\ln (1+t)} & \\
\Phi_{5}(t)=e^{t}-t-1 . &
\end{array}
$$

We observe that $\Phi_{1}$ and $\Phi_{2}$ satisfy the $\Delta^{\prime}$-condition globally in $[0,+\infty)$; moreover $\Phi_{1}$ and $\Phi_{2}$ belong to the class $\triangle_{2} \cap \nabla_{2}$ globally in $[0,+\infty)$. The function $\Phi_{3}$ satisfy $\Delta^{\prime}$-condition for all $t \geq t_{0}$ but $\Phi_{3} \notin \nabla_{2}$. The function $\Phi_{5} \in \nabla_{2}$ but $\Phi_{5} \notin \Delta_{2}$. Finally $\Phi_{4} \in \Delta_{2} \cap \nabla_{2}$ but $\Phi_{4} \notin \Delta^{\prime}$. For further details refer to (Adams, 1975; Dall'Aglio, Mascolo, \& Papi, 1997, 1998; Klimov, 2000; Krasnosel'skij, 1961; Mascolo \& Papi, 1996; Moscariello \& Nania, 1991; Rao $\&$ Ren, 1991). Now we can introduce Orlicz spaces and Orlicz Sobolev Spaces, $L^{\Phi}$ and $W^{1} L^{\Phi}$. Let $\Omega \subseteq \mathbb{R}^{N}$ be a bounded and open set, the Orlicz class $K^{\Phi}(\Omega)$ is the set of all measurable functions $u: \Omega \rightarrow \mathbb{R}$ (equivalence classes modulo equality $\mathcal{L}^{N}$ a.e. in $\Omega$ ) satisfying $\int_{\Omega} \Phi(|u|) d \mathcal{L}^{N}<+\infty$. The Orlicz space $L^{\Phi}(\Omega)$ is defined to be the linear hull of $K^{\Phi}(\Omega)$, thus it consists of all measurable functions $u$ such that $\lambda u \in K^{\Phi}(\Omega)$ for some $\lambda>0$. Moreover, the equality $K^{\Phi}(\Omega) \equiv L^{\Phi}(\Omega)$ holds if and only if $\Phi \in \Delta_{2}$.

Definition 10 If $\Omega \subset \mathbb{R}^{N}$ is a bounded open set and $\Phi \in \Delta_{2}$ then we define

$$
W^{1} L^{\Phi}(\Omega)=\left\{u \in L^{\Phi}(\Omega): \partial_{i} u \in L^{\Phi}(\Omega) \text { for } i=1, \ldots, N\right\}
$$

where $\partial_{i} u$ are the weak derivatives of $u$ for $i=1, \ldots, N$. 
Theorem 2 Let $\Phi \in \triangle_{2}$, then $L^{\Phi}(\Omega)$ and $W^{1} L^{\Phi}(\Omega)$ are Banach spaces with the following norms

$$
\|u\|_{\Phi, \Omega}=\inf \left(k>0: \int_{\Omega} \Phi\left(\frac{|u|}{k}\right) d \mathcal{L}^{N} \leq 1\right)
$$

and

$$
\|u\|_{1, \Phi, \Omega}=\|u\|_{\Phi, \Omega}+\sum_{i=1}^{N}\left\|\partial_{i} u\right\|_{\Phi, \Omega} .
$$

For greater details we refer to (Adams, 1975; Dall'Aglio, Mascolo, \& Papi, 1997, 1998; Klimov, 2000; Krasnosel'skij, 1961; Mascolo \& Papi, 1996; Moscariello \& Nania, 1991; Rao \& Ren, 1991).

Let $\Phi$ be a $\mathrm{N}$-function then there exists a real valued function $p$ defined on $[0,+\infty)$ and having the following properties: $p(0)=0, p(t)>0$ if $t>0, p$ is increasing and right continuous on $(0,+\infty)$ such that

$$
\Phi(t)=\int_{0}^{t} p(s) d s \quad \text { for every } t \in(0,+\infty)
$$

and

$$
\dot{\Phi}_{+}(t)=p(t) \quad \text { a.e. on }(0,+\infty) .
$$

Definition 11 Let $p$ be a real valued function defined on $[0,+\infty)$ and having the following properties: $p(0)=0$, $p(t)>0$ if $t>0, p$ is increasing and right continuous on $(0,+\infty)$. We define

$$
\widetilde{p}(s)=\sup _{p(t) \leq s}(t)
$$

and

$$
\widetilde{\Phi}(t)=\int_{0}^{t} \widetilde{p}(s) d s .
$$

The N-functions $\Phi$ and $\widetilde{\Phi}$ are complementary N-functions.

Particularly from the relationship (2.1) of the Definition 2 we get the following Young inequality

$$
a b \leq \widetilde{\Phi}(a)+\Phi(b) .
$$

We now recall the notion of Sobolev conjugate of a $\mathrm{N}$-function. For a sake of simplicity, we will only consider the case of a function in $\triangle_{2}^{m}$.

Definition 12 Assume that $\Phi \in \Delta_{2}^{m}$, with $1 \leq m<N$. We define the Sobolev conjugate of $\Phi$ as the function $\Phi^{*}$ whose inverse is defined by

$$
\left(\Phi^{*}\right)^{-1}(t)=\int_{0}^{t} \frac{\Phi^{-1}(s)}{s^{1+\frac{1}{N}}} d s .
$$

Remark 12 Using condition (ii) of Lemma 1 we have $\Phi^{-1}(s)<c s^{\frac{1}{m}}$ for $0<s<1$, then the integral in (2.9) is finite and it is easy to check that $\Phi^{*}$ is a N-function. If $\Phi(t)=t^{m}$, with $1 \leq m<N$, then $\Phi^{*}(t)=\left(\frac{t}{m^{*}}\right)^{m^{*}}$ where $m^{*}=\frac{m N}{N-m}$ is the Sobolev conjugate exponent of $m$. Moreover, if $\Phi(t)$ is equivalent near infinity to $t^{m} \ln ^{a}(1+t)$, with $1 \leq m<N$ and $a>0$, then $\Phi^{*}(t)$ is equivalent near infinity to $t^{m^{*}}(\ln (1+t))^{\frac{a N}{N-m}}$.

Lemma 3 Let $\Phi$ be a $N$-function in $\triangle_{2}^{m} \cap \nabla_{2}^{r}$ with $1 \leq r \leq m<N$, then $\Phi^{*} \in \Delta_{2}^{m^{*}} \cap \nabla_{2}^{r^{*}}$.

\section{Local Boundedness}

Let $E$ be a $\mathcal{L}^{N}$-measurable subset of $\mathbb{R}^{N}$, then with $|E|$ we denote the $\mathcal{L}^{N}$-measure of $E$. If $u \in W_{l o c}^{1} L^{\Phi}(\Omega), k$ is a real number and $Q_{R}\left(x_{0}\right)$ is a cube strictly contained in $\Omega$ we set

$$
\begin{aligned}
& A(k, R)=\left\{x \in Q_{R}: u(x)>k\right\}=\{u>k\} \cap Q_{R}, \\
& B(k, R)=\left\{x \in Q_{R}: u(x)<k\right\}=\{u<k\} \cap Q_{R} .
\end{aligned}
$$


Remark 13 We have $|A(k, R)|=\left|Q_{R}\right|-|B(k, R)|$ for almost every $k \in \mathbb{R}$, so that when necessary we can assume without loss of generality that all the vcalues $k$ under consideration will satisfy this relation.

Our proof is based on the following Caccioppoli inequalities.

Theorem 3 Let $u \in V$ be a local minimizer of $\mathcal{F}[u, \Omega]$, with $f$ satisfying conditions $H_{1, f}, H_{2,1, f}$ or $H_{1, f}, H_{2,2, f}$; then there exist two real positive constants $C_{1, C a c}, C_{2, \text { Cac }}$ depending only on $c_{1}, c_{2}, N, p$ and $\beta$ such that for every $x_{0} \in \Omega$, for every cube $Q_{\varrho}\left(x_{0}\right) \subset \subset Q_{R}\left(x_{0}\right) \subset \subset \Omega$ and for every $k \in \mathbb{R}$ we have

$$
\int_{A(k, \varrho)} \Phi(|\nabla u|) d \mathcal{L}^{N} \leq \frac{C_{1, C a c}}{(R-\varrho)^{\beta p^{*}}} \int_{A(k, R)}\left(\Phi^{*}(u-k)\right)^{\beta} d \mathcal{L}^{N}+C_{2, C a c}\left(\Phi^{*}(k)\right)^{\beta}|A(k, R)|
$$

and

$$
\int_{B(k, \varrho)} \Phi(|\nabla u|) d \mathcal{L}^{N} \leq \frac{C_{1, C a c}}{(R-\varrho)^{\beta p^{*}}} \int_{B(k, R)}\left(\Phi^{*}(k-u)\right)^{\beta} d \mathcal{L}^{N}+C_{2, C a c}\left(\Phi^{*}(k)\right)^{\beta}|B(k, R)| .
$$

Proof. It follows by Theorem 4.1 of Dall'Aglio, Mascolo, and Papi (1997).

For more details see Dall'Aglio, Mascolo, and Papi (1997, 1998).

Remark 14 Let us take $v=u+1$ and $h=k+1$, then using (3.1) and (3.2) we get

$$
\int_{A(h, \varrho)} \Phi(|\nabla v|) d \mathcal{L}^{N} \leq \frac{H_{1}}{(R-\varrho)^{\beta p^{*}}} \int_{A(h, R)}\left(\Phi^{*}(v-h)\right)^{\beta} d \mathcal{L}^{N}+H_{2}\left(\Phi^{*}(h)\right)^{\beta}|A(h, R)|
$$

and

$$
\int_{B(h, \varrho)} \Phi(|\nabla v|) d \mathcal{L}^{N} \leq \frac{H_{1}}{(R-\varrho)^{\beta p^{*}}} \int_{B(h, R)}\left(\Phi^{*}(h-v)\right)^{\beta} d \mathcal{L}^{N}+H_{2}\left(\Phi^{*}(h)\right)^{\beta}|B(h, R)| .
$$

Using an abuse of notation we will always identify $u$ with $v$ and $h$ with $k$.

We can now introduce the adequate De Giorgi classes related to the functional (1.1).

Definition 13 Let $u \in W_{l o c}^{1,1}(\Omega)$; we say that $u \in D G_{\Phi, \Phi^{* \beta}}^{+}\left(\Omega, H_{1}, H_{2}, R_{0}, k_{0}\right)$ if for all couple of concentric cubes $Q_{\varrho} \subset Q_{R} \subset Q_{R_{0}} \Subset \Omega$, with $\varrho<R<R_{0}$, and for all $k \geq k_{0} \geq 0$ we have

$$
\int_{A(k, \varrho)} \Phi(|\nabla u|) d \mathcal{L}^{N} \leq \frac{H_{1}}{(R-\varrho)^{\beta p^{*}}} \int_{A(k, R)}\left(\Phi^{*}(u-k)\right)^{\beta} d \mathcal{L}^{N}+H_{2}\left(\Phi^{*}(k)\right)^{\beta}|A(k, R)| .
$$

Definition 14 Let $u \in W_{l o c}^{1,1}(\Omega)$; we say that $u \in D G_{\Phi, \Phi^{* \beta}}^{-}\left(\Omega, H_{1}, H_{2}, R_{0}, k_{0}\right)$ if for all couple of concentric cubes $Q_{\varrho} \subset Q_{R} \subset Q_{R_{0}} \Subset \Omega$, with $\varrho<R<R_{0}$, and for all $k \leq-k_{0} \leq 0$ we have

$$
\int_{B(k, \varrho)} \Phi(|\nabla u|) d \mathcal{L}^{N} \leq \frac{H_{1}}{(R-\varrho)^{\beta p^{*}}} \int_{B(k, R)}\left(\Phi^{*}(k-u)\right)^{\beta} d \mathcal{L}^{N}+H_{2}\left(\Phi^{*}(k)\right)^{\beta}|B(k, R)| .
$$

Definition 15 Let $u \in W_{l o c}^{1,1}(\Omega)$; we say that $u \in D G_{\Phi, \Phi^{* \beta}}\left(\Omega, H_{1}, H_{2}, R_{0}, k_{0}\right)$ if $u \in D G_{\Phi, \Phi^{* \beta}}^{ \pm}\left(\Omega, H_{1}, H_{2}, R_{0}, k_{0}\right)$, that is

$$
D G_{\Phi, \Phi^{* \beta}}\left(\Omega, H_{1}, H_{2}, R_{0}, k_{0}\right)=D G_{\Phi, \Phi^{* \beta}}^{+}\left(\Omega, H_{1}, H_{2}, R_{0}, k_{0}\right) \cap D G_{\Phi, \Phi^{* \beta}}^{-}\left(\Omega, H_{1}, H_{2}, R_{0}, k_{0}\right) .
$$

Lemma 4 Let $\theta>0$ and let $\left\{x_{i}\right\}_{i \in \mathbb{N}}$ be a sequence of real positive numbers such that

$$
x_{i+1} \leq C B^{i} x_{i}^{1+\theta}
$$

with $C>0$ and $B>1$. If $x_{0} \leq C^{-\frac{1}{\theta}} B^{-\frac{1}{\theta^{2}}}$ then we get

$$
x_{i} \leq C^{-\frac{1}{\theta}} x_{0}
$$

and

$$
\lim _{i \rightarrow+\infty} x_{i}=0 .
$$


Theorem 4 Let $u \in V$ be a local minimizer of $\mathcal{F}[u, \Omega]$, with $f$ satisfying conditions $H_{0, f}, H_{1, f}, H_{2, f}, H_{3,1, f}$ or $H_{0, f}, H_{1, f}, H_{2, f}, H_{3,2, f}$; then $u$ is locally bounded in $\Omega$ and there exists a $R_{0}>0$ such that for every $x_{0} \in \Omega$ and $0<R \leq \min \left(R_{0}, \operatorname{dist}\left(x_{0}, \partial \Omega\right)\right)$ we have

$$
\Phi^{*}\left(\operatorname{ess}-\sup _{Q_{\frac{R}{2}}}(|u|)\right) \leq 1+C(R)\left[\int_{Q_{R}} \Phi^{*}(|u|)^{\beta} d \mathcal{L}^{N}\right]^{\frac{r^{*}-m}{m(1-\beta)}}
$$

where $C(R)$ is a real positive constant depending on $R, N, m, r$ and $\beta$.

Proof. It follows by Theorem 3.1 of Dall'Aglio, Mascolo, and Papi (1997).

For more details see Dall'Aglio, Mascolo, and Papi (1997, 1998).

Now we introduce our first result that improves the precedent theorem shown in general in (Dall' Aglio, Mascolo, \& Papi, 1997, 1998).

Theorem 5 Let $u \in V$ be a local minimizer of $\mathcal{F}[u, \Omega]$, with $f$ satisfying conditions $H_{0, f}, H_{1, f}, H_{2, f}, H_{3,1, f}$ or $H_{0, f}$, $H_{1, f}, H_{2, f}, H_{3,2, f}$; then if $\left|k_{0}\right|+\sup (u) \leq M$ we have

$$
\left(\Phi^{*}\left(e s s-\sup _{Q_{\frac{R}{2}}}(u)\right)\right)^{\beta} \leq \frac{C_{22}\left|A\left(k_{0}, R\right)\right|^{\vartheta}}{R^{\frac{m^{*} \beta-\gamma \alpha_{2}}{\alpha_{1}}}} \int_{A\left(k_{0}, R\right)}\left(\Phi^{*}\left(u-k_{0}\right)\right)^{\beta} d \mathcal{L}^{N}+\Phi_{*, \beta}^{-1}\left(R^{m^{*} \beta}\right)
$$

where $\alpha_{1}=\frac{[N-(1-\beta) m] r-N m \beta}{(N-m) r}, \alpha_{2}=\frac{\beta m N}{(N-m) r}-1, \gamma=N-m^{*} \beta, \vartheta$ is the positive solution of $\vartheta(\vartheta+1)=\alpha_{1}$, i.e. $\vartheta=\frac{-1+\sqrt{1+4 \alpha_{1}}}{2}$, and $C_{22}$ is a real positive number depending on $N, \beta, \alpha_{1}, \alpha_{2}$ and $\gamma$.

Proof. For $\frac{R}{2} \leq \sigma<\tau \leq R$ let $\eta(x)$ be a function of class $C_{c}^{\infty}\left(Q_{\frac{\tau+\sigma}{2}}\right)$ with $\eta=1$ on $Q_{\sigma}$ and $|\nabla \eta| \leq \frac{c}{\tau-\sigma}$. Setting $k>h>k_{0}, \zeta=\eta(u-k)_{+}$we have

$$
\int_{A(k, \sigma)}\left(\Phi^{*}(u-k)\right)^{\beta} d \mathcal{L}^{N} \leq \int_{E_{\zeta}}\left(\Phi^{*}(\zeta)\right)^{\beta} d \mathcal{L}^{N}
$$

where $E_{\zeta}=\operatorname{Supp}(\zeta) \subset A\left(k, \frac{\tau+\sigma}{2}\right)$. Since

$$
\int_{E_{\zeta}}\left(\Phi^{*}(\zeta)\right)^{\beta} d \mathcal{L}^{N} \leq \int_{E_{\zeta}}\left(\Phi^{*}\left(\zeta+t_{0}\right)\right)^{\beta} d \mathcal{L}^{N} \leq c \int_{E_{\zeta}}\left(\zeta+t_{0}\right)^{m^{*} \beta} d \mathcal{L}^{N}
$$

using the Sobolev Inequality we get

$$
\begin{aligned}
\int_{E_{\zeta}}\left(\zeta+t_{0}\right)^{m^{*} \beta} d \mathcal{L}^{N} & =\int_{Q_{\frac{\tau+\sigma}{2}}}\left(\zeta+t_{0}\right)^{m^{*} \beta} d \mathcal{L}^{N} \\
& \leq c_{N, m, \beta}\left[\int_{Q_{\frac{\tau+\sigma}{2}}}|\nabla \zeta|^{\frac{\beta m N}{N-(1-\beta) m}} d \mathcal{L}^{N}\right]^{\frac{N-(1-\beta) m}{N-m}} \\
& =c_{N, m, \beta}\left[\int_{E_{\zeta}}|\nabla \zeta|^{\frac{\beta m n N}{N-(1-\beta) m}} d \mathcal{L}^{N}\right]^{\frac{N-(1-\beta) m}{N-m}}
\end{aligned}
$$

Since $\beta<\frac{r(N-m)}{m(N-r)}$ using the Hölder Inequality it follows

$$
\int_{E_{\zeta}}|\nabla \zeta|^{\frac{\beta m N}{N-(1-\beta) m}} d \mathcal{L}^{N} \leq\left|E_{\zeta}\right|^{1-\frac{N m \beta}{(N-(1-\beta) m) r}}\left[\int_{E_{\zeta}}|\nabla \zeta|^{r} d \mathcal{L}^{N}\right]^{\frac{N m \beta}{N-(1-\beta) m) r}}
$$

and

$$
\int_{E_{\zeta}}\left(g^{*}(\zeta)\right)^{\beta} d \mathcal{L}^{N} \leq c_{N, p, \beta}\left|E_{\zeta}\right|^{\alpha_{1}}\left[\int_{E_{\zeta}}|\nabla \zeta|^{r} d \mathcal{L}^{N}\right]^{\frac{\beta m n}{(N-m) r}}
$$


where $\alpha_{1}=\frac{[N-(1-\beta) m] r-N m \beta}{(N-m) r}$. Since $\beta>\frac{(N-m) r}{m N}$ then $\frac{\beta m N}{(N-m) r}>1$ and

$$
\int_{E_{\zeta}}\left(g^{*}(\zeta)\right)^{\beta} d \mathcal{L}^{N} \leq c_{N, p, \beta}\left|E_{\zeta}\right|^{\alpha_{1}} \int_{E_{\zeta}}|\nabla \zeta|^{r} d \mathcal{L}^{N}\left[\int_{E_{\zeta}}|\nabla \zeta|^{r} d \mathcal{L}^{N}\right]^{\alpha_{2}}
$$

where $\alpha_{2}=\frac{\beta m N}{(N-m) r}-1$. Since $t^{r} \leq \Phi(t)\left[\right.$ Refer $\left.H_{2, f}\right]$ we have

$$
\int_{E_{\zeta}}|\nabla \zeta|^{r} d \mathcal{L}^{N} \leq \int_{A\left(k, \frac{\tau+\sigma}{2}\right)} \Phi(|\nabla u|) d \mathcal{L}^{N}+c\left[\int_{A\left(k, \frac{\tau+\sigma}{2}\right)}\left(\Phi^{*}\left(\frac{u-k}{\tau-\sigma}\right)\right)^{\beta} d \mathcal{L}^{N}+\left|A\left(k, \frac{\tau+\sigma}{2}\right)\right|\right] .
$$

Using the Caccioppoli inequality we obtain

$$
\int_{E_{\zeta}}|\nabla \zeta|^{r} d \mathcal{L}^{N} \leq c^{\Upsilon} \Upsilon(u)
$$

where

$$
\Upsilon(u)=C_{1, C a c} \int_{A(k, \tau)}\left(\Phi^{*}\left(\frac{u-k}{\tau-\sigma}\right)\right)^{\beta} d \mathcal{L}^{N}+C_{2, C a c}\left(\left(\Phi^{*}(k)\right)^{\beta}+1\right)|A(k, \tau)| .
$$

From (3.14) and (3.15) it follows

$$
\int_{E_{\zeta}}\left(\Phi^{*}(\zeta)\right)^{\beta} d \mathcal{L}^{N} \leq c_{N, p, \beta}\left|E_{\zeta}\right|^{\alpha_{1}}[\Upsilon(u)][\Upsilon(u)]^{\alpha_{2}} .
$$

Let $M>d>\left(\Phi^{*}\right)^{-1}\left(R^{m^{*}}\right)$ be a constant that we shall fix later, and define

$$
\begin{aligned}
& k_{0}=d \\
& k_{i+1}=k_{i}+\Phi_{*, \beta}^{-1}\left(\frac{\Phi_{* \beta}(d)}{2^{i m}}\right) \quad \text { for } i \geq 1 ; \\
& r_{i}=\frac{R}{2}\left(1+2^{-i}\right) ; \\
& u_{i}=U\left(k_{i}, r_{i}\right) ;
\end{aligned}
$$

where $\Phi_{*, \beta}(t)=\left(\Phi^{*}(t)\right)^{\beta}$, then, using (3.16), we get

$$
\int_{A_{i+1}}\left(\Phi^{*}\left(u-k_{i+1}\right)\right)^{\beta} d \mathcal{L}^{N} \leq c_{N, p, \beta}\left|A_{i}\right|^{\alpha_{1}}\left[U\left(k_{i}, r_{i}\right)\right] \cdot\left[U\left(k_{i}, r_{i}\right)\right]^{\alpha_{2}} .
$$

where

$$
\left\{\begin{array}{l}
A_{i}=A\left(k_{i}, r_{i}\right) \\
U\left(k_{i}, r_{i}\right)=C_{1, C a c} \int_{A\left(k_{i}, r_{i}\right)}\left(\Phi^{*}\left(\frac{u-k_{i}}{r_{i}-r_{i+1}}\right)\right)^{\beta} d \mathcal{L}^{N}+C_{2, C a c}\left(\left(\Phi^{*}\left(k_{i}\right)\right)^{\beta}+1\right)\left|A\left(k_{i}, r_{i}\right)\right|
\end{array}\right.
$$

Now we give two alternative estimates of

$$
U\left(k_{i}, r_{i}\right)=C_{1, C a c} \int_{A\left(k_{i}, r_{i}\right)}\left(\Phi^{*}\left(\frac{u-k_{i}}{r_{i}-r_{i+1}}\right)\right)^{\beta} d \mathcal{L}^{N}+C_{2, C a c}\left(\left(\Phi^{*}\left(k_{i}\right)\right)^{\beta}+1\right)\left|A\left(k_{i}, r_{i}\right)\right| .
$$

Since $u$ is bounded we get

$$
U\left(k_{i}, r_{i}\right) \leq \frac{C_{1, C a c} 2^{m^{+} \beta} 2^{m^{+} \beta i}}{R^{m^{+} \beta}} R^{N}\left(\Phi^{*}(M)\right)^{\beta}+C_{2, C a c}\left(\left(\Phi^{*}(M)\right)^{\beta}+1\right) R^{N}
$$

then

$$
U\left(k_{i}, r_{i}\right) \leq G_{M} 2^{m^{+} \beta i} R^{\gamma}
$$


where $G_{M}=2^{m^{+} \beta}\left(C_{1, C a c}+C_{2, C a c}\right)\left(\left(\Phi^{*}(M)\right)^{\beta}+1\right), C_{C a c}=\left(C_{1, C a c}+C_{2, C a c}\right)$ and $\gamma=N-m^{*} \beta$, since $\frac{N-m}{m}>\beta$ then $\gamma>0$.

Moreover, since

$$
\left|A\left(k_{i}, r_{i}\right)\right| \leq \frac{1}{\left(\Phi^{*}\left(k_{i+1}-k_{i}\right)\right)^{\beta}} \int_{A\left(k_{i}, r_{i}\right)}\left(\Phi^{*}\left(u-k_{i}\right)\right)^{\beta} d \mathcal{L}^{N}
$$

we get

$$
U\left(k_{i}, r_{i}\right) \leq\left[\frac{C_{1, C a c}}{\left(r_{i}-r_{i+1}\right)^{m^{*} \beta}}+\frac{C_{2, C a c}\left(\left(g^{*}\left(k_{i}\right)\right)^{\beta}+1\right)}{\left(\Phi^{*}\left(k_{i}-k_{i}\right)\right)^{\beta}}\right] \int_{A\left(k_{i}, r_{i}\right)}\left(\Phi^{*}\left(u-k_{i}\right)\right)^{\beta} d \mathcal{L}^{N} ;
$$

since $k_{i}=d+\sum_{j=1}^{i} \Phi_{*, \beta}^{-1}\left(\frac{\Phi_{* \beta}(d)}{2^{m^{+} \beta j}}\right) \leq d+\sum_{j=1}^{i} \frac{d}{2^{m^{+} \beta j}} \leq 2^{m^{+} \beta} d$ and $\left(\Phi^{*}(d)\right)^{\beta}>R^{m^{*} \beta}$, it follows

$$
\begin{aligned}
U\left(k_{i}, r_{i}\right) & \leq\left[\frac{C_{1, C a c} m^{m^{*} \beta} 2^{m^{*} \beta i}}{R^{m^{*} \beta}}+\frac{C_{2, C a c}\left(2^{m^{+} \beta}\right)^{m^{+} \beta} 2^{m^{*} \beta}}{R^{m^{*} \beta} \beta}\right]_{A\left(k_{i}, r_{i}\right)}\left(\Phi^{*}\left(u-k_{i}\right)\right)^{\beta} d \mathcal{L}^{N} \\
& \leq C_{C a c} C_{2, m, N} \frac{2^{m^{*} \beta i}}{R^{m^{*} \beta}} \int_{A\left(k_{i}, r_{i}\right)}\left(\Phi^{*}\left(u-k_{i}\right)\right)^{\beta} d \mathcal{L}^{N} .
\end{aligned}
$$

Using (3.18), (3.19) and (3.20) it follows

$$
\begin{aligned}
\int_{A_{i+1}}\left(\Phi^{*}\left(u-k_{i+1}\right)\right)^{\beta} d \mathcal{L}^{N} \leq & c_{N, p, \beta}\left|A_{i}\right|^{\alpha_{1}} \cdot\left[G_{M} 2^{m^{+} \beta i} R^{\gamma}\right]^{\alpha_{2}} . \\
& \cdot\left[C_{C a c} C_{2, m, N} \frac{2^{m^{*} \beta i}}{R^{m^{*} \beta}} \int_{A\left(k_{i}, r_{i}\right)}\left(\Phi^{*}\left(u-k_{i}\right)\right)^{\beta} d \mathcal{L}^{N}\right] .
\end{aligned}
$$

Since $\left|A\left(k_{i}, r_{i}\right)\right| \leq \frac{1}{\left(\Phi^{*}\left(k_{i+1}-k_{i}\right)\right)^{\beta}} \int_{A\left(k_{i}, r_{i}\right)}\left(\Phi^{*}\left(u-k_{i}\right)\right)^{\beta} d \mathcal{L}^{N}$ we have

$$
\int_{A_{i+1}}\left(\Phi^{*}\left(u-k_{i+1}\right)\right)^{\beta} d \mathcal{L}^{N} \leq \frac{C_{2, m, N, M, \beta}}{\left(\left(\Phi^{*}(d)\right)^{\beta}\right)^{\alpha_{1}}} \frac{2^{m^{*} \beta\left(1+\alpha_{1}+\alpha_{2}\right) i}}{R^{m^{*} \beta-\gamma \alpha_{2}}} \cdot\left[\int_{\left(k_{i}, r_{i}\right)}\left(\Phi^{*}\left(u-k_{i}\right)\right)^{\beta} d \mathcal{L}^{N}\right]^{1+\alpha_{1}}
$$

and

$$
\omega_{i+1} \leq \frac{C_{2, m, N, M, \beta}}{R^{m^{*} \beta-\gamma \alpha_{2}}\left(\left(g^{*}(d)\right)^{\beta}\right)^{\alpha_{1}}} 2^{m^{*} \beta\left(1+\alpha_{1}+\alpha_{2}\right) i} \omega_{i}^{1+\alpha_{1}}
$$

where $\omega_{i}=\left|A\left(k_{i}, r_{i}\right)\right|^{\vartheta} \int_{A\left(k_{i}, r_{i}\right)}\left(\Phi^{*}\left(u-k_{i}\right)\right)^{\beta} d \mathcal{L}^{N}$ and $\vartheta$ is the positive solution of $\vartheta(\vartheta+1)=\alpha_{1}$.

Choosing $d$ such that

$$
\varpi_{0} \leq\left(\frac{C_{2, m, N, M, \beta}}{R^{m^{*} \beta-\gamma \alpha_{2}}\left(\left(\Phi^{*}(d)\right)^{\beta}\right)^{\alpha_{1}}}\right)^{-\frac{1}{\alpha_{1}}} 2^{-\frac{m^{*} \beta\left(1+\alpha_{1}+\alpha_{2}\right)}{\alpha_{1}^{2}}}
$$

we can apply Lemma 4; then

$$
\lim _{i \rightarrow+\infty} \varpi_{i}=0
$$

and

$$
U\left(d, \frac{R}{2}\right)=0
$$

The condition imposed on $d$ will be satisfied taking

$$
\left(\Phi^{*}(d)\right)^{\beta}=\frac{\left(C_{2, m, N, M, \beta}\right)^{\frac{1}{\alpha_{1}}} 2^{\frac{m^{*} \beta\left(1+\alpha_{1}+\alpha_{2}\right)}{\alpha_{1}^{2}}}}{R^{\frac{m^{*} \beta-\gamma \alpha_{2}}{\alpha_{1}}}} \varpi_{0}+\Phi_{*, \beta}^{-1}\left(R^{m^{*} \beta}\right)
$$


hence we have

$$
\left(\Phi^{*}\left(\text { ess }-\sup _{Q_{\frac{R}{2}}}(u)\right)\right)^{\beta} \leq \frac{C\left|A\left(k_{0}, R\right)\right|^{\vartheta}}{R^{\frac{m^{*} \beta-\gamma \alpha_{2}}{\alpha_{1}}}} \int_{A\left(k_{0}, R\right)}\left(\Phi^{*}\left(u-k_{0}\right)\right)^{\beta} d \mathcal{L}^{N}+\Phi_{*, \beta}^{-1}\left(R^{m^{*} \beta}\right)
$$

where $C=\left(C_{2, m, N, M, \beta}\right)^{\frac{1}{\alpha_{1}}} 2^{\frac{m^{*} \beta\left(1+\alpha_{1}+\alpha_{2}\right)}{\alpha_{1}^{2}}}$ and $\vartheta$ is the positive solution of $\vartheta(\vartheta+1)=\alpha_{1}$, i.e $\vartheta=\frac{-1+\sqrt{1+4 \alpha_{1}}}{2}$.

\section{Proof of the Main Theorem}

Lemma 5 Let $u \in V$ be a local minimizer of $\mathcal{F}[u, \Omega]$, with $f$ satisfying conditions $H_{0, f}, H_{1, f}, H_{2, f}, H_{3,1, f}$ or $H_{0, f}$, $H_{1, f}, H_{2, f}, H_{3,2, f}$, and let $2 k_{0}=M(2 R)-m(2 R)$. Assume that $\left|A\left(k_{0}, R\right)\right| \leq \gamma\left|Q_{R}\right|$ for some $\gamma<1$. If for an integer $v$, it holds that

$$
\operatorname{osc}(u, 2 R) \geq 2^{v+1} \Phi_{*, \beta}^{-1}\left(R^{m^{*} \beta}\right)
$$

where $\Phi_{*, \beta}^{-1}(t)$ is the inverse of $\Phi_{*, \beta}(t)=\left(\Phi^{*}(t)\right)^{\beta}$, then, setting $k_{\nu}=M(2 R)-2^{-v-1} \operatorname{osc}(u, 2 R)$ we have

$$
\left|A\left(k_{v}, R\right)\right| \leq c_{N, m, \beta, M} \frac{R^{N \alpha_{1}+\left(N-m^{*} \beta\right)\left(1+\alpha_{2}\right)}}{\nu^{\alpha_{1}}}
$$

where $\alpha_{1}=\frac{[N-(1-\beta) m] r-N m \beta}{(N-m) r}, \alpha_{2}=\frac{\beta m N}{(N-m) r}-1$ are the constants of Theorem 5.

Proof. For $k_{0}<h<k$ let us define

$$
v(x)= \begin{cases}k-h & \text { if } u \geq k \\ u-h & \text { if } h<u<k \\ 0 & \text { if } u \leq h .\end{cases}
$$

We have $v=0$ in $Q_{R} \backslash A\left(k_{0}, R\right)$ and since $\left|Q_{R} \backslash A\left(k_{0}, R\right)\right| \geq(1-\gamma)\left|Q_{R}\right|$ by Sobolev inequality we get

$$
\begin{aligned}
\int_{A(k, R)}\left(\Phi^{*}(v)\right)^{\beta} d \mathcal{L}^{N} & \leq \int_{A(k, R)}\left(\Phi^{*}\left(v+t_{0}\right)\right)^{\beta} d \mathcal{L}^{N} \\
& \leq \int_{A(k, R)}\left(v+t_{0}\right)^{m^{*} \beta} d \mathcal{L}^{N} \\
& \leq c_{N, m, \beta}\left[\int_{\Delta_{R}}|\nabla u|^{\frac{\beta m N}{N-(1-\beta) m}} d \mathcal{L}^{N}\right]^{\frac{N-(1-\beta) m}{N-m}} c_{N, p, \beta}\left|\Delta_{R}\right|^{\alpha_{1}}\left[\int_{\Delta_{R}}|\nabla u|^{r} d \mathcal{L}^{N}\right]^{\frac{\beta m N}{(N-m) r}}
\end{aligned}
$$

where $\Delta_{R, k, h}=A(k, R)-A(h, R)$ and $\alpha_{1}=\frac{[N-(1-\beta) m] r-N m \beta}{(N-m) r}$. Since $t^{r} \leq g(t)$ and $\beta>\frac{(N-m) r}{m N}$ then $\frac{\beta m N}{(N-m) r}>1$ and we get

$$
\int_{A(k, R)}\left(\Phi^{*}(v)\right)^{\beta} d \mathcal{L}^{N} \leq c_{N, p, \beta}\left|\Delta_{R}\right|^{\alpha_{1}}\left[\int_{\Delta_{R}} \Phi(|\nabla u|) d \mathcal{L}^{N}\right]\left[\int_{\Delta_{R}} \Phi(|\nabla u|) d \mathcal{L}^{N}\right]^{\alpha_{2}} .
$$

where $\alpha_{2}=\frac{\beta m N}{(N-m) r}-1$. Since for $h \leq k_{v}$ we have $M(2 R)-h \geq M(2 R)-k_{v} \geq 2^{-v-1} \operatorname{osc}(u, 2 R) \geq \Phi_{*, \beta}^{-1}\left(R^{m^{*} \beta}\right)$ then, using Caccioppoli inequality (3.1) for the levels $k=k_{i}=M(2 R)-2^{-i-1} o s c(u, 2 R)$ and $h=k_{i-1}$, proceeding as in the previous Theorem 5 we get

$$
\left|A\left(k_{\nu}, R\right)\right|^{\frac{1}{\alpha_{1}}} \leq\left(c_{N, m, \beta, M}\right)^{\frac{1}{\alpha_{1}}}\left|\Delta_{R, i}\right| R^{\frac{\left(N-m^{*} \beta\right)\left(1+\alpha_{2}\right)}{\alpha_{1}}} .
$$

Summing over $i$ from 1 to $v$ we have

$$
v\left|A\left(k_{v}, R\right)\right|^{\frac{1}{\alpha_{1}}} \leq\left(c_{N, m, \beta, M}\right)^{\frac{1}{\alpha_{1}}} R^{N} R^{\frac{\left(N-m^{*} \beta\right)\left(1+\alpha_{2}\right)}{\alpha_{1}}}
$$

and

$$
\left|A\left(k_{\nu}, R\right)\right| \leq c_{N, m, \beta, M} \frac{R^{N \alpha_{1}+\left(N-m^{*} \beta\right)\left(1+\alpha_{2}\right)}}{\gamma^{\alpha_{1}}} .
$$

Lemma 6 Let $\varphi$ be a positive function, and assume that there exist a constant $q$ and a number $\tau, 0<\tau<1$ such that for every $R<R_{0}$

$$
\varphi(\tau R) \leq \tau^{\delta} \varphi(R)+B R^{\varkappa}
$$


with $0<\varkappa<\delta$ and

$$
\varphi(t) \leq q \varphi\left(\tau^{k} R\right)
$$

for every $t \in\left(\tau^{k+1} R, \tau^{k} R\right)$. Then for every $\varrho<R \leq R_{0}$ we have

$$
\varphi(\varrho) \leq C\left\{\left(\frac{\varrho}{R}\right)^{\varkappa} \varphi(R)+B \varrho^{\varkappa}\right\}
$$

where $C$ is a constant depending only on $q, \tau, \varkappa$ and $\delta$.

Now we can prove our main Theorem.

Proof. (Proof of Theorem 1) Let $k_{0}=\frac{M(2 R)+m(2 R)}{2}$ we can assume $\left|A\left(k_{0}, R\right)\right| \leq \frac{1}{2}\left|Q_{R}\right|$ since otherwise we can assume $\left|B\left(k_{0}, R\right)\right|=\left|Q_{R}\right|-\left|A\left(k_{0}, R\right)\right| \leq \frac{1}{2}\left|Q_{R}\right|$ and it will be sufficient to write $-u$ instead of $u$. Setting $k_{v}=$ $M(2 R)-2^{-v-1} \operatorname{osc}(u, 2 R)$ we have $k_{v}>k_{0}$ and

$$
\begin{aligned}
\left(\Phi^{*}\left(\operatorname{ess}-\sup _{Q_{\frac{R}{2}}}\left(u-k_{v}\right)\right)\right)^{\beta} & \leq \frac{C}{R^{\frac{m^{*} \beta-\gamma \alpha_{2}}{\alpha_{1}}}}\left|A\left(k_{\nu}, R\right)\right|^{\vartheta} \int_{A\left(k_{0}, R\right)}\left(\Phi^{*}\left(u-k_{\nu}\right)\right)^{\beta} d \mathcal{L}^{N}++\Phi_{*, \beta}^{-1}\left(R^{m^{*} \beta}\right) \\
& \leq \frac{C}{R^{\frac{m^{*} \beta-\gamma \alpha_{2}}{\alpha_{1}}}}\left|A\left(k_{v}, R\right)\right|^{\vartheta} R^{N}\left(\Phi^{*}\left(\operatorname{ess}-\sup _{Q_{R}}\left(u-k_{v}\right)\right)\right)^{\beta}++\Phi_{*, \beta}^{-1}\left(R^{m^{*} \beta}\right) .
\end{aligned}
$$

Let us choose an integer $v$ such that

$$
c\left(\frac{c_{N, m, \beta, M}}{v^{\alpha_{1}}}\right)^{\vartheta}<\frac{1}{2^{\varkappa}}
$$

If $\operatorname{osc}(u, 2 R) \geq 2^{\nu+1} \Phi_{*, \beta}^{-1}\left(R^{m^{*} \beta}\right)$ then by Lemma 5 we get

$$
\left(\Phi^{*}\left(e s s-\sup _{Q_{\frac{R}{2}}}\left(u-k_{v}\right)\right)\right)^{\beta} \leq \frac{1}{2^{\varkappa}} R^{\Lambda}\left(\Phi^{*}\left(e s s-\sup _{Q_{R}}\left(u-k_{v}\right)\right)\right)^{\beta}+\Phi_{*, \beta}^{-1}\left(R^{m^{*} \beta}\right)
$$

where $\Lambda=N+\left(N \alpha_{1}+\left(N-m^{*} \beta\right)\left(1+\alpha_{2}\right)\right) \vartheta-\frac{m^{*} \beta-\gamma \alpha_{2}}{\alpha_{1}}$. Since $\Lambda \geq 0$ [refer $\mathrm{H}_{1, f}$ and (1.3)] it follows

$$
\left(\Phi^{*}\left(e s s-\sup _{Q_{\frac{R}{2}}}\left(u-k_{v}\right)\right)\right)^{\beta} \leq \frac{1}{2^{\varkappa}}\left(\Phi^{*}\left(e s s-\sup _{Q_{R}}\left(u-k_{\nu}\right)\right)\right)^{\beta}+\Phi_{*, \beta}^{-1}\left(R^{m^{*} \beta}\right)
$$

and

$$
\operatorname{osc}\left(u, \frac{R}{2}\right) \leq\left(1-\frac{1}{2^{v+2}}\right) \operatorname{osc}(u, R)+\chi R .
$$

In conclusion, either $\operatorname{osc}(u, 2 R) \leq 2^{v+1} \Phi_{*, \beta}^{-1}\left(R^{m^{*} \beta}\right)$ or, if $\operatorname{osc}(u, 2 R) \geq 2^{v+1} \Phi_{*, \beta}^{-1}\left(R^{m^{*} \beta}\right), \operatorname{osc}\left(u, \frac{R}{2}\right) \leq\left(1-\frac{1}{2^{v+2}}\right)$ $\operatorname{osc}(u, R)+\chi R$, in any case we get

$$
\operatorname{osc}\left(u, \frac{R}{2}\right) \leq\left(1-\frac{1}{2^{v+2}}\right) \operatorname{osc}(u, R)+\chi R^{\omega}
$$

with $0<\omega<1$. Apply Lemma 6 with $\tau=\frac{1}{4}$ and $\delta=\log _{\frac{1}{4}}\left(1-\frac{1}{2^{x+2}}\right)$ and $\omega<\delta$ we have

$$
\operatorname{osc}(u, \varrho) \leq C\left\{\left(\frac{\varrho}{R}\right)^{\omega} \operatorname{osc}(u, R)+B \varrho^{\omega}\right\}
$$

for every $\varrho<R \leq \min \left(R_{0}, \operatorname{dist}\left(x_{0}, \partial \Omega\right)\right)$.

\section{Appendix: Hypotheses on $\beta$}

Let us consider the system

$$
\left\{\begin{array}{l}
1 \leq r \leq m<N \\
\frac{r(N-m)}{m N}<\beta<\min \left(\frac{r(N-m)}{m(N-r)}, \frac{N-m}{m}\right) \\
\alpha_{1} N+\left(N-m^{*} \beta\right)\left(1+\alpha_{2}\right) \leq N \\
\alpha_{1} N+\left[\alpha_{1} N+\left(N-m^{*} \beta\right)\left(1+\alpha_{2}\right)\right] \vartheta \alpha_{1}-m^{*} \beta+\left(N-m^{*} \beta\right) \alpha_{2} \geq 0
\end{array}\right.
$$


where $\alpha_{1}=\frac{[N-(1-\beta) m] r-N m \beta}{(N-m) r}, \alpha_{2}=\frac{\beta m N}{(N-m) r}-1, m^{*}=\frac{m N}{N-m}$ and $\vartheta$ is the positive solution of $\vartheta(\vartheta+1)=\alpha_{1}$, i.e. $\vartheta=\frac{-1+\sqrt{1+4 \alpha_{1}}}{2}$. Moreover, since $\frac{r(N-m)}{m(N-r)}<1$ then we get $\min \left(\frac{r(N-m)}{m(N-r)}, \frac{N-m}{m}\right)<1$. Let us take

$$
\begin{aligned}
\alpha_{1} & =\frac{[N-(1-\beta) m] r-N m \beta}{(N-m) r} \\
& =\frac{(N-m) r-m \beta(N-r)}{(N-m) r} \\
& =1-\frac{N-r}{r} \frac{m}{N-m} \beta \\
& =1-\frac{m^{*}}{r^{*}} \beta \\
\alpha_{2} & =\frac{\beta m N}{(N-m) r}-1 \\
& =\frac{m^{*}}{r} \beta-1 \\
\vartheta & =\frac{-1+\sqrt{1+4 \alpha_{1}}}{2} \\
& =\frac{-1+\sqrt{1+4\left(1-\frac{m^{*}}{r^{*}} \beta\right)}}{2} \\
& =\frac{-1+\sqrt{5-4 \frac{m^{*}}{r^{*}} \beta}}{2}
\end{aligned}
$$

and

$$
\begin{aligned}
m^{*}=N \frac{x}{1-x} & \text { where } x=\frac{m}{N} \\
r^{*}=N \frac{y}{1-y} & \text { where } y=\frac{r}{N} \\
\frac{m^{*}}{r^{*}}=\frac{x(1-y)}{y(1-x)} & \beta=z \\
\frac{m^{*}}{r}=\frac{x}{y(1-x)} &
\end{aligned}
$$

then the system (5.1) can be written in this way

$$
\left\{\begin{array}{l}
\frac{1}{N} \leq y \leq x \leq 1 \\
\frac{y(1-x)}{x}<z<\min \left(\frac{y(1-x)}{x(1-y)}, \frac{1-x}{x}\right) \\
\left(1-\frac{x z}{1-x}\right) \frac{x z}{y(1-x)}<\frac{x(1-y)}{y(1-x)} z \\
T(x, y, z) \geq 0
\end{array}\right.
$$

where

$$
\begin{aligned}
T(x, y, z)= & {\left[1-\frac{x(1-y)}{y(1-x)} z+\left(1-\frac{x z}{1-x}\right) \frac{x z}{y(1-x)}\right]\left(\frac{-1+\sqrt{5-4 \frac{x(1-y)}{y(1-x)}}}{2}\right)\left(1-\frac{x(1-y)}{y(1-x)} z\right)-} \\
& -\frac{x z}{1-x}+\left(1-\frac{x z}{1-x}\right)\left(\frac{x z}{y(1-x)}-1\right)+1-\frac{x(1-y)}{y(1-x)} z
\end{aligned}
$$

If

$$
w=w(x, z)=\frac{x z}{1-x}
$$

we get

$$
\left\{\begin{array}{l}
\frac{1}{N} \leq y \leq x \leq 1 \\
y<w<\min \left(\frac{y}{(1-y)}, 1\right) \\
(1-w) \frac{w}{y}<\frac{(1-y)}{y} w \\
M(y, w) \geq 0
\end{array}\right.
$$

where

$$
\begin{aligned}
M(y, w)= & {\left[1-\frac{(1-y)}{y} w+(1-w) \frac{w}{y}\right]\left(\frac{\left.-1+\sqrt{5-4 \frac{(1-y)}{y}}\right)}{2}\right)\left(1-\frac{(1-y)}{y} w\right)-} \\
& -w+(1-w)\left(\frac{w}{y}-1\right)+1-\frac{(1-y)}{y} w
\end{aligned}
$$


With simple algebraic calculations it follows that the system (5.1) is equivalent to the system

$$
\left\{\begin{array} { l } 
{ \frac { 1 } { N } \leq y \leq x \leq 1 } \\
{ y < w < \operatorname { m i n } ( \frac { y } { ( 1 - y ) } , 1 ) } \\
{ ( 1 - w ) \frac { w } { y } < \frac { ( 1 - y ) } { y } w \Longleftrightarrow y < w } \\
{ E ( y , w ) \geq 0 }
\end{array} \Leftrightarrow \left\{\begin{array}{l}
\frac{1}{N} \leq y \leq x \leq 1 \\
y<w<\min \left(\frac{y}{(1-y)}, 1\right) \\
E(y, w) \geq 0
\end{array}\right.\right.
$$

where

$$
\begin{aligned}
E(y, w)= & {[y-(1-y) w+(1-w) w]\left(\frac{-1+\sqrt{5-4 \frac{(1-y)}{y} w}}{2}\right)\left(1-\frac{(1-y)}{y} w\right)-} \\
& -w y+(1-w)(w-y)+y-(1-y) w .
\end{aligned}
$$

Since

$$
\begin{array}{ll}
e_{1}(y)=E(y, y)=y^{2}\left(\frac{-1+\sqrt{1+4 y}}{2}\right) \geq 0 & \text { for every } 0 \leq y \leq 1 \\
e_{2}(y)=E\left(y, \frac{y}{1-y}\right)=-\frac{2 y^{3}}{1-y} \leq 0 & \text { for every } 0 \leq y<1 . \\
e_{3}(y)=E(y, 1)=y-1+\frac{(2 y-1)^{2}}{y}\left(\frac{-1+\sqrt{5-4 \frac{(1-y)}{y}}}{2}\right) & \text { for every } \frac{1}{2} \leq y<1
\end{array}
$$

and

for every $w, y \in(0,1)$, where

$$
\frac{\partial E}{\partial y}=w+G(y, w) \geq 0
$$

$$
\begin{aligned}
G(y, w)= & (1+w)\left(\frac{-1+\sqrt{5-4 \frac{(1-y)}{y} w}}{2}\right)\left(1-\frac{(1-y)}{y} w\right)+ \\
& +[y-(1-y) w+(1-w) w]\left(\frac{-1+\sqrt{5-4 \frac{(1-y)}{y} w}}{2}\right)\left(\frac{w}{y^{2}}\right)+ \\
& +[y-(1-y) w+(1-w) w]\left(1-\frac{(1-y)}{y} w\right) \frac{w}{y^{2} \sqrt{5-4 \frac{(1-y)}{y} w} \geq 0} \\
& \text { for every } w, y \in(0,1) .
\end{aligned}
$$

using the Bolzano-Weiestrass Teoremand the Implicit Theorem we get that the equation

$$
E(y, w)=0
$$

implicitly defines a fuction

$$
w=\tilde{w}(y)
$$

for every $0<y<1$. Moreover the system

$$
\left\{\begin{array}{l}
\frac{1}{N} \leq y \leq x \leq 1 \\
y<w<\min \left(\frac{y}{(1-y)}, 1\right) \\
E(y, w) \geq 0
\end{array}\right.
$$

has as solutions the region defined by

$$
y<w<\bar{w}_{y}
$$

where $\bar{w}_{y}$ is the function defined by

$$
\bar{w}_{y}=\min (\tilde{w}(y), 1)
$$

for every $0<y<1$. With a simple algebraic calculations we get the following relations

$$
\left\{\begin{array}{l}
1 \leq r \leq m \leq N \\
\frac{r}{m^{*}}<\beta<\frac{N}{m^{*}} \bar{r}_{r, N} .
\end{array}\right.
$$


Let us take

$$
\bar{\beta}_{r, m, N}=\frac{N}{m^{*}} \bar{w}_{r, N}
$$

then we have the following hypoteses on $\beta$

$$
\left\{\begin{array}{l}
1 \leq r \leq m \leq N \\
\frac{r}{m^{*}}<\beta<\bar{\beta}_{r, m, N} .
\end{array}\right.
$$

In the following figures we draw the graphs of $E(y, w), e_{1}(y), e_{2}(y)$ and $e_{3}(y)$.
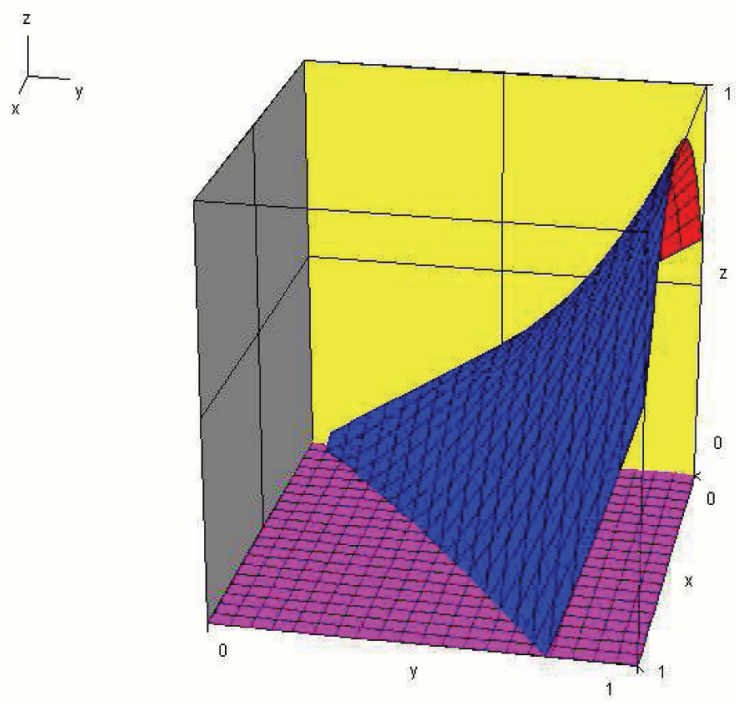

Figure 1. The graphs of $E(y, w)$

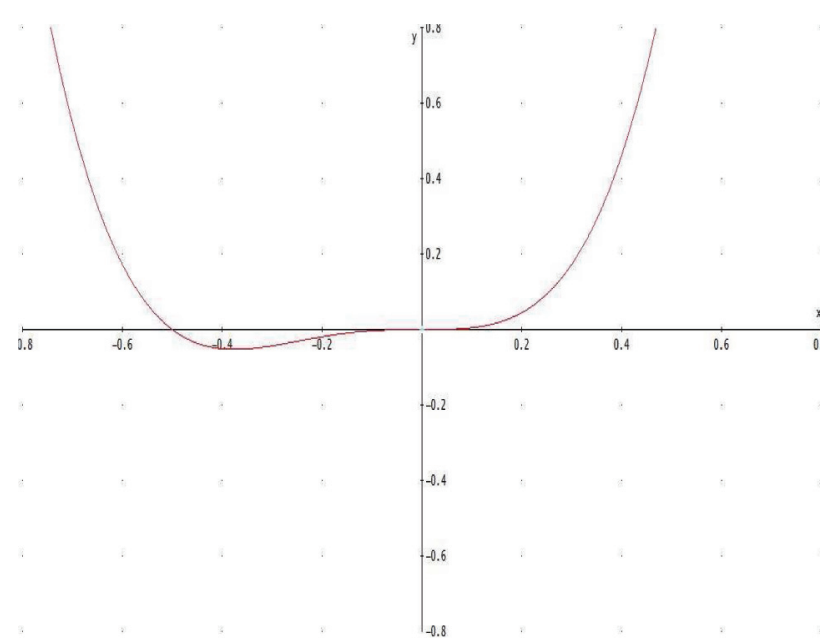

Figure 2. The graphs of $e_{1}(y)$ 


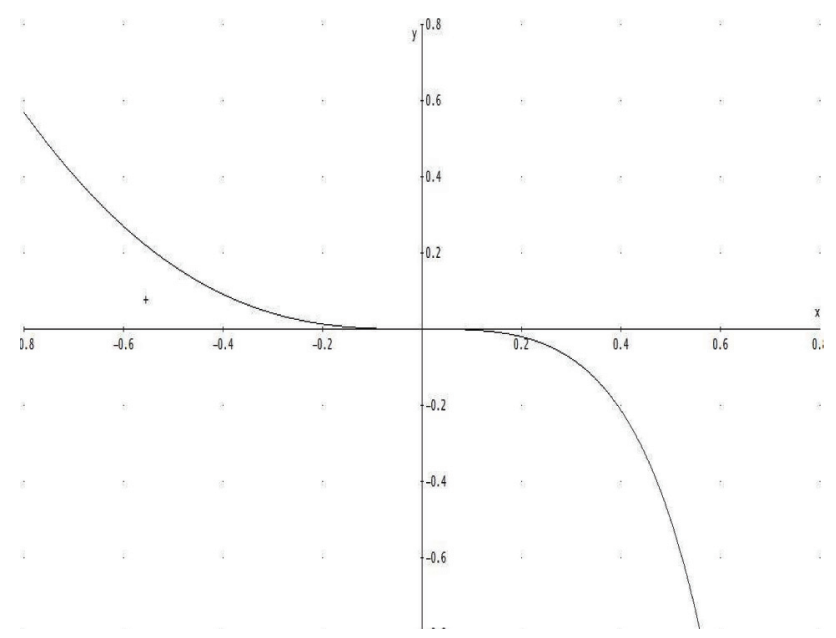

Figure 3. The graphs of $e_{2}(y)$

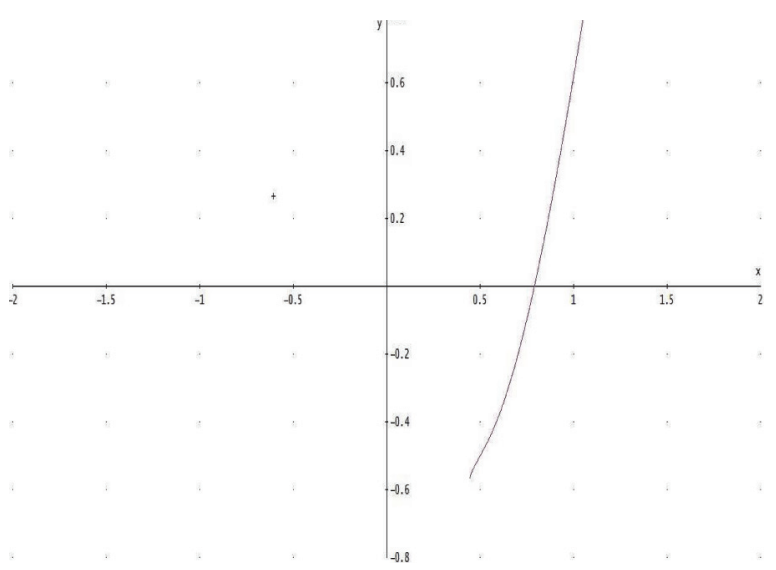

Figure 4. The graphs of $e_{3}(y)$

\section{References}

Adams, R. (1975). Sobolev spaces. New York: Academic Press.

Dall'Aglio, A., Mascolo, E., \& Papi, G. (1997). Local boundedness for minima of functionals with nonstandard growth conditions. Quaderni del Dipartimento di Matematica "U. Dini", 7.

Dall'Aglio, A., Mascolo, E., \& Papi, G. (1998). Regularity for local minima of functionals with nonstandard growth conditions. Rend. Mat., 18(VII), 305-326.

De Giorgi, E. (1957). Sulla differenziabilità e l'analicità delle estremali degli integrali multipli, Mem. Accad. Sci Torino, cl. Sci. Fis. Mat. Nat., 3, 25-43.

Giaquinta, M., \& Giusti, E. (1982). On the regularity of minima of variational integrals. Acta Math., 148, 31-46. http://dx.doi.org/10.1007/BF02392725

Giaquinta, M., \& Giusti, E. (1984). Quasi-minima. Ann. Inst. H. Poincarè (Analyse non lineaire), 1 , 79-107.

Giusti, E. (1994). Metodi diretti nel Calcolo delle Variazioni, U. M. I., Bologna.

Gosez, J. P. (1974). Nonlinear elliptic problems for equations with rapidly (or slowly) increasing coefficents. Trans. Amer. Math. Soc., 190, 163-205. http://dx.doi.org/10.1090/S0002-9947-1974-0342854-2

Granucci, T. (2006). Osservazioni sulla continuità hölderiana per un minimo di un funzionale convesso con crescite nonstandard. Rend. dell'Istit. Mat. dell'Univ. di Trieste, 37, 21-43.

Granucci, T. (2014a). Hölder continuity for scalar minimizers of integals of the Calculus of Variations with general growths. Afr. Mat., 25(1), 197-212. http://dx.doi.org/10.1007/s13370-012-0109-3

Granucci, T. (2014b). Remarks on the Harnack inequality of local-minima of scalar integral functionals with 
general growth conditions, J. Appl. Math. Phys., 2, 194-203.

Granucci, T. (submitted). Remarks on the continuity of the local minimizer of scalar integral functionals with nonstandard growth conditions, forcaming on Nolinear Analysis and Differential Equations.

Klimov, V. S. (2000). Embedding Theorems and Continuity of Generalized Solutions of Quasilinear Elliptic Equations. Diff. Equations, 36(6), 870-877, translated from Diff. Uravn.,36(6), 784-791.

Krasnosel'skij, M. A., \& Rutickii, Ya. B. (1961). Convex Functions and Orlicz Spaces. Groningen: Noordhoff.

Lieberman, G. M. (1991). The Natural Generalization of the Natural Conditions of Ladyzhenskaya and Ural'tseva for Elliptic Equations. Comm. Part. Diff. Equat., 16, 331-361. http://dx.doi.org/10.1080/03605309108820761

Marcellini, P. (1993). Regularity for elliptic equations with general growth conditions. J. Diff. Eq., 2(105), 296333. http://dx.doi.org/10.1006/jdeq.1993.1091

Marcellini, P. (1996). Regularity for some scalar variational problems under general growth. J. Optim. Theory Apll., 1, 161-181. http://dx.doi.org/10.1007/BF02192251

Mascolo, E., \& Papi, G. (1994). Local Bounddeness of Minimizers of Integrals of the Calculus of Variations. Ann. Mat. Pura Appl., 167, 323-339. http://dx.doi.org/10.1007/BF01760338

Mascolo, E., \& Papi, G. (1996). Harnack inequality for minimizer of integral functionals with general growth conditions. Nonlin. Diff. Eq. Appl., 3, 231-244. http://dx.doi.org/10.1007/BF01195916

Moscariello, G., \& Nania, L. (1991). Hölder continuity of minimizers of functionals with nonstandard growth conditions. Ricerche di Matematica, 15(2), 259-273.

Nash, J. (1958). Continuity of solutions of parabolic and elliptic differential equations. Amer. Journal of Math., 80, 931-953. http://dx.doi.org/10.2307/2372841

Rao, M. M., \& Ren, Z. D. (1991). Theory of Orlicz spaces. New Work: Marcel Dekker.

\section{Copyrights}

Copyright for this article is retained by the author(s), with first publication rights granted to the journal.

This is an open-access article distributed under the terms and conditions of the Creative Commons Attribution license (http://creativecommons.org/licenses/by/3.0/). 